\title{
Implicaciones de los actores locales en las intervenciones para la promoción de la salud bucodental destinadas a niños de edad preescolar y escolar en entornos vulnerables
}

\author{
Involvement of local stakeholders in oral health promotion interventions for pre-school and school chil- \\ dren in vulnerable settings
}

\section{Señor Editor:}

En dos artículos previamente publicados en esta revista, unos investigadores reconocen la importancia de involucrar a los padres y a los profesores en las intervenciones para la promoción de la salud bucodental (PSBD) destinadas a niños de edad preescolar y escolar, que provienen de entornos vulnerables, para reducir la prevalencia y la gravedad de las caries en esta población ${ }^{(1,2)}$. Estamos de acuerdo con la afirmación de esos autores.

Sin embargo, pensamos que sería necesario tener un enfoque intersectorial más integral, que incluyan otros actores locales, como líderes y autoridades locales. Los resultados de una investigación que hemos realizado recientemente en la región del Cusco, demuestran la necesidad de un liderazgo por parte de actores locales para la implementación de las intervenciones de PSBD, destinadas a los alumnos que viven en un entorno vulnerable con la finalidad de mejorar la eficiencia y la perennidad y continuidad de las intervenciones ${ }^{(3)}$.

Se debería movilizar a esos actores locales en forma sostenida, para la adaptación y el desarrollo de la intervención de PSBD, pues su implicación es una buena ocasión para mejorar la comprensión de las intervenciones de PSBD en entornos vulnerables y marginados. Por cierto, diferentes elementos del contexto especifico al entorno en donde se implementan las intervenciones de PSBD influyen en su eficacia ${ }^{(4)}$. Además, la implicación de actores locales puede por supuesto brindar un mejor conocimiento de los desafíos y de las dinámicas presentes ${ }^{(5,6)}$. Tal proceso permite también estar más enfocado en las necesidades locales ${ }^{(7)}$, darle localmente mayor credibilidad a las intervenciones ${ }^{(5,8)}$, y desarrollar intervenciones mejor contextualizadas en las realidades lugareñas ${ }^{(5,6)}$.

Sin embargo, se deben abordar ciertos desafíos para facilitar su implicación y así mejorar el estado de salud bucodental de niños de edad preescolar y escolar.

\footnotetext{
Departamento de Enfermería, Université du Québec à Rimouski. Rimouski, Quebec, Canadá.

2 Departamento de Medicina Familiar y Medicina de Emergencia, Facultad de Medicina y Ciencias de la Salud, Université de Sherbrooke. Longueuil, Quebec, Canadá.

3 Clínica Odontológica "Alina Rodríguez de Gómez", Universidad San Antonio Abad del Cusco. Cusco, Perú.

${ }^{4}$ Carrera Profesional de Odontología, Universidad Nacional San Antonio Abad del Cusco. Cusco, Perú.

a Enf., PhD; Profesor.

b $\mathrm{CD}, \mathrm{MSc}$; Profesor; Director de la Clinica Odontológica

PhD; Profesora.
} 
Es también importante recordar que la implicación de actores locales y el poder que se les facilita son a menudo simbólicos y los intentos de implementación de las iniciativas en las que participan carecen con frecuencia de continuidad, lo que puede generar cansancio y desconfianza en las comunidades destinatarias ${ }^{(9)}$.

Un primer desafío sería de asegurar una participación plena de los actores locales en distintas etapas del proceso de implementación de una intervención de PSBD. Para superar este primer desafío, sería necesario desarrollar herramientas de animación e intercambio que asegurarán su participación plena ${ }^{(10)}$, y limitar los desequilibrios de poder entre los responsables de la toma de decisión, profesionales y actores locales ${ }^{(7,10)}$. El desarrollo de un lenguaje común sin terminología compleja permitiría asimismo minimizar los malentendidos entre los actores y favorecer los intercambios ${ }^{(11)}$.

Un segundo desafío sería de mantener el contacto con los actores locales ${ }^{(8)}$. Para facilitar el mantenimiento del contacto, ciertos autores dan prioridad a la contratación y a la formación de un personal local que tiene como propósito de asegurar el seguimiento de la intervención y mantener intercambios periódicos con los actores locales ${ }^{(11)}$.

Un último desafío consistiría en garantizar que este proceso fuera acompañado de acciones concretas para las comunidades locales, con el afán de no contribuir a las inequidades ${ }^{(12)}$, ni aumentar la desconfianza ${ }^{(9)}$. Entre estas acciones, por lo menos habría que abordar ciertas preocupaciones de las comunidades vulnerables como el acceso al agua potable ${ }^{(13)}$, el acceso y el costo de los productos de higiene bucal y de los alimentos frescos (14) así como elevar el nivel de competencia de los actores involucrados ${ }^{(8,12)}$.

$\mathrm{Al}$ concluir, estamos convencidos de que es necesario incrementar el compromiso de los actores locales para llevar a cabo las intervenciones de PSBD destinadas a niños de edad preescolar y escolar procedentes de un entorno vulnerable. Tal proceso estimulará el surgimiento de intervenciones de PSBD mejor coordinadas y adaptadas a las realidades locales, que por ende permitirán bonificar los efectos de las intervenciones de PSBD con los niños para mejorar su estado de salud bucodental y general.

Financiamiento: Estos trabajos de reflexión han recibido un subsidio de los Institutos de investigación en salud de Canadá, de las becas del Fondo de investi- gación de Quebec en salud, del ministerio de la Enseñanza superior, de la Investigación y de la Ciencia de Quebec y de la Facultad de medicina y de las ciencias de la salud de la Universidad de Sherbrooke.

Conflicto de intereses: Los autores declaran no tener ningún conflicto de intereses.

\author{
Dave A. Bergeron 1,2,a \\ Fernando Murillo ${ }^{3,4, b}$ \\ Isabelle Gaboury ${ }^{2, c}$
}

\section{Correspondencia: \\ Dave A. Bergeron}

300 Allée des Ursulines

Rimouski (Quebec), Canadá

G5L 3A1

Correo electrónico: Dave_Bergeron@uqar.ca

\section{REFERENCIAS BIBLIOGRÁFICAS}

1. Sanchez-Vilchez A, Sihuay-Torres K. Estrategias para el abordaje de caries dental en preescolares. Rev Estomatol Herediana. 2019;29(3):247-8.

2. Carrasco-Loyola M, Orejuela-Ramirez F. Consecuencias clínicas de caries dental no tratada en preescolares y escolares de instituciones educativas públicas. Rev Estomatol Herediana. 2018;28(4):223-8.

3. Bergeron DA, Talbot LR, Gaboury I. Intersectoral oral health promotion interventions for schoolchildren living in remote rural Andean communities: a realist evaluation. Global Health Promot. 2019; OnlineFirst. doi: $10.1177 / 1757975919870160$

4. Albino J, Tiwari T. Preventing childhood caries: a review of recent behavioral research. J Dent Res. 2016;95(1):35-42.

5. Siddiqui FR. Annotated bibliography on participatory consultations to help aid the inclusion of marginalized perspectives in setting policy agendas. Int J Equity Health. 2014;13(1):124.

6. Puertas B, Schlesser M. Assessing community health among indigenous populations in Ecuador with a participatory approach: Implications for health reform. J Community Health. 2001;26(2):133-47.

7. Taylor A. Getting it right: Culturally safe approaches to health partnership work in low to middle income countries. Nurse Educ Pract. 2017;24:49-54.

8. Bonevski B, Randell M, Paul C, et al. Reaching the hard-to-reach: a systematic review of strategies for improving health and medical research with socially 
disadvantaged groups. BMC Med Res Methodol. 2014;14(1):42.

9. Attree P, French B, Milton B, Povall S, Whitehead M, Popay J. The experience of community engagement for individuals: a rapid review of evidence. Health Soc Care Community. 2011;19(3):250-60.

10. Bernier J. La recherche partenariale comme espace de soutien à l'innovation. Global Health Promot. 2014;21(S1):58-63.

11. Kyoon-Achan G, Lavoie J, Avery Kinew K, et al. Innovating for Transformation in First Nations Health Using Community-Based Participatory Research. Qual Health Res. 2018;28(7):1036-49.
12. Labonte R, Spiegel J. Setting global health research priorities: burden of disease and inherently global health issues should both be considered. Br Med J. 2003;326(7392):722.

13. Moynihan P, Makino Y, Petersen PE, Ogawa H. Implications of WHO Guideline on Sugars for dental health professionals. Community Dent Oral Epidemiol. 2018;46(1):1-7.

14. Abadía-Barrero CE, Martínez-Parra AG. Care and consumption: A Latin American social medicine's conceptual framework to comprehend oral health inequalities. Glob Public Health. 2016;12(10):1228-41.

Recibido: 15-12-2019 